\title{
INDEX OF SUBJECTS.
}

Air, see Carbon-monoxide, Nickel carbonyl

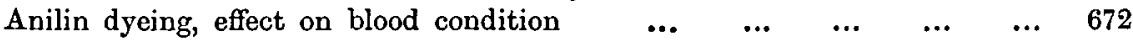

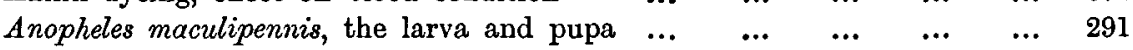

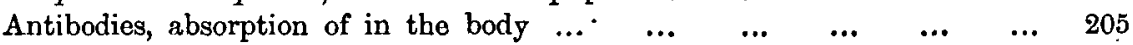

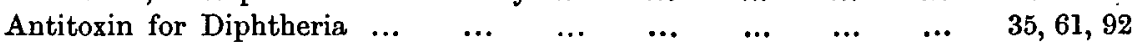

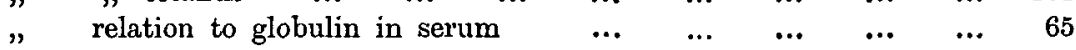

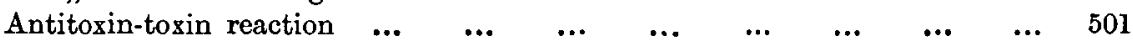

$\begin{array}{llllllllll}\text { Bacillus ceylanensis ... } & \ldots & \ldots & \ldots & \ldots & \ldots & \ldots & \ldots & \ldots & 8\end{array}$

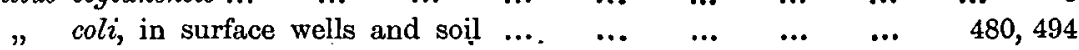

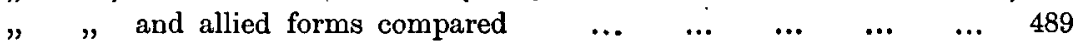

$\begin{array}{lllllllll} & \text { diphtherice in milk } \quad \ldots & \ldots & \ldots & \ldots & \ldots & \ldots & \ldots & 32\end{array}$

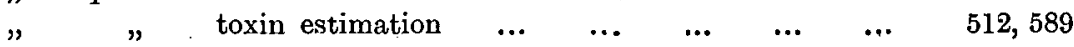

$"$ " see Diphtheria

pestis, see Plague bacillus

Benzidine colour-therapy, sẹe Trypanosoma

Beri-beri, see Ship

Blood, see Anilin, Antibodies, Antitoxin, Cattle-plague, Carbon monoxide, Nickel carbonyl, Nitro-benzine

$\begin{array}{lllllllll}\text { Bordet-Gengou reaction in Yaws ... } & \ldots & \ldots & \ldots & \ldots & \ldots & \ldots & 565\end{array}$

Carbon monoxide, toxicology of $\ldots \begin{array}{llllllll}\ldots & \ldots & \ldots & \ldots & \ldots & \ldots & \ldots & 528\end{array}$

Cattle-plague; filtration of blood in $\begin{array}{llllllll}\ldots & \ldots & \ldots & \ldots & \ldots & \ldots & 570\end{array}$

Cerebro-spinal Meningitis, see Meningococcus

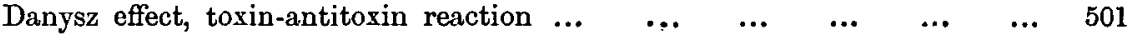

$\begin{array}{llllllll}\text { Diphtheria antitoxin } & \ldots & \ldots & \ldots & \ldots & \ldots & \ldots & \ldots 35,61,65,92\end{array}$

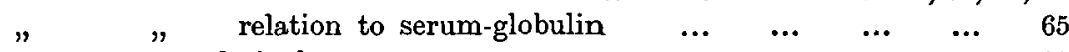

$\begin{array}{llllllllll} & \text { post-scarlatinal } & \ldots & \ldots & \ldots & \ldots & \ldots & \ldots & \ldots & 593\end{array}$

$" \quad$ see Bacillus

Dog, see Piroplasma

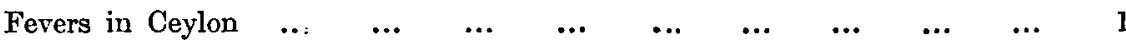

Flea, see under Plague

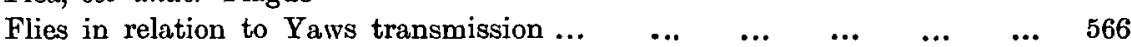

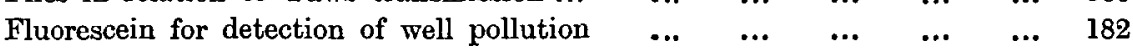

Fowls, see Ship-beri-beri

$\begin{array}{lllllllll}\text { Framboesia tropica (Yaws) } & \ldots & \ldots & \ldots & \ldots & \ldots & \ldots & \ldots & 558\end{array}$ 


\section{Index of Subjects}

PAGE
Guinea-pigs, variation in weight ...

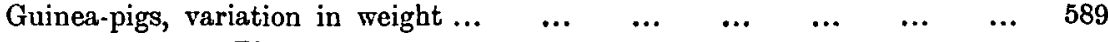
" see Plague

$\begin{array}{llllllllll}\text { Immunity in Yaws } \ldots & \ldots & \ldots & \ldots & \ldots & \ldots & \ldots & \ldots & \ldots & 565\end{array}$

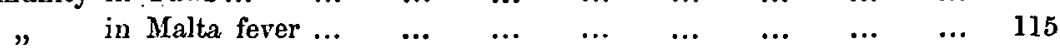

" see Antibodies, Antitoxin, Bordet, Light, Leucocytosis, Supersensitation

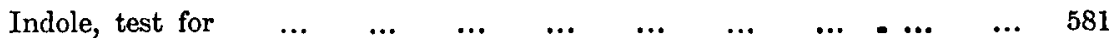

Insects, see Anopheles, Flea, Flies

Leucocytosis during immunisation with Diphtheria toxin $\quad \ldots \quad$...

Light, effect on vaccinia in animals $\begin{array}{llllllll}. . & \ldots & \ldots & \ldots & \ldots & \ldots & \ldots & 155\end{array}$

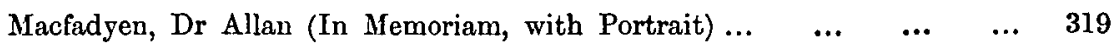

Malarial mosquito, see Anopheles

$\begin{array}{lllllllll}\text { Malta fever, vaccine treatment } & \ldots & \ldots & \ldots & \ldots & \ldots & \ldots & \ldots & 115\end{array}$

Mediterranean fever, see Malta

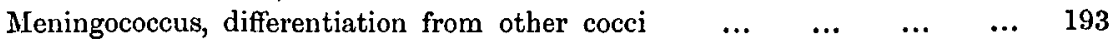

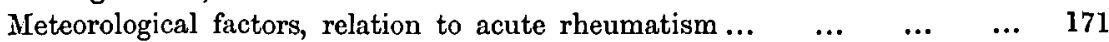

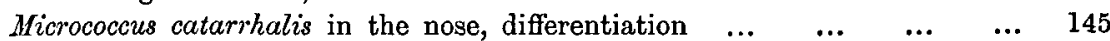

$" \quad$ intracellularis, see Meningococcus

" melitensis, see Malta

$\begin{array}{lllllllllll}" \text { neoformans } & \ldots & \ldots & \ldots & \ldots & \ldots & \ldots & \ldots & \ldots & 13\end{array}$

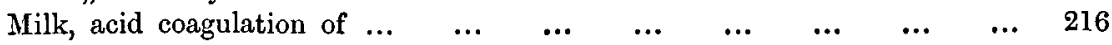

$\begin{array}{llllllllll}\# \text { bacteriology of } & \ldots & \ldots & \ldots & \ldots & \ldots & \ldots & \ldots & \ldots & 22\end{array}$

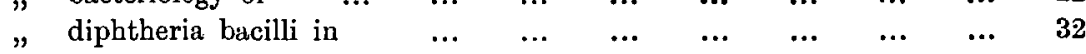

Monkey, see Yaws, Syphilis

Mosquito, see Anopheles

Mouse, see Trypanosoma

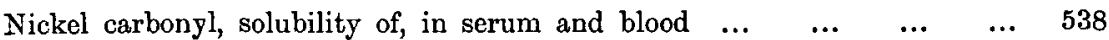

$\begin{array}{lllllllllll} & \| & \text { toxicology of } & \ldots & \ldots & \ldots & \ldots & \ldots & \ldots & \ldots & 525\end{array}$

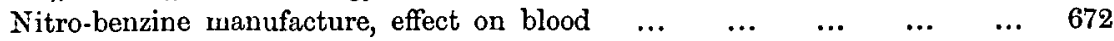

Parakeet, see Protozoal disease of

Pigeons, see Ship-beri-beri

$\begin{array}{llllllllllll}\text { Piroplasma canis } & \ldots & \ldots & \ldots & \ldots & \ldots & \ldots & \ldots & \ldots & \ldots & 232\end{array}$

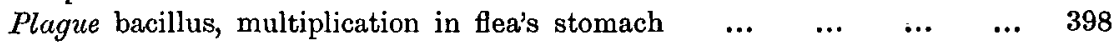

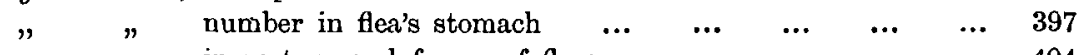

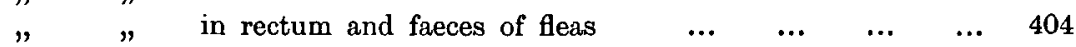

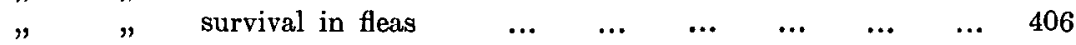

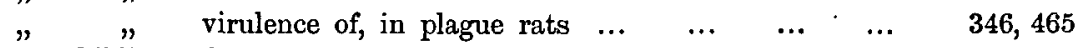

$\begin{array}{lllllllllll} & \text { bibliography } & \ldots & \ldots & \ldots & \ldots & \ldots & \ldots & \ldots & \ldots & 720\end{array}$

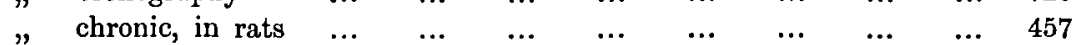

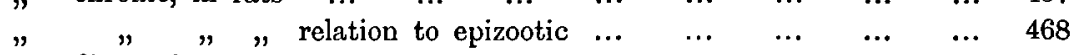

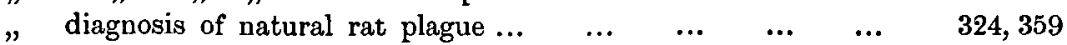

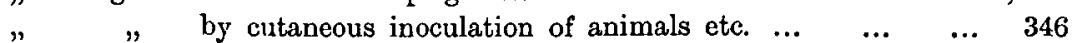

$\begin{array}{llllllll} & \text { in domestic animals other than rats } & \ldots & \ldots & \ldots & \ldots & \ldots & 891\end{array}$

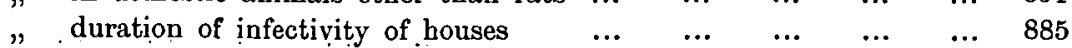


Plague dwellings, see influence of

epidemics, experimental in anis 


\section{Index of Subjects}

Plague observations on, in Punjab villages (Dhand, Kasel)
$"$
$"$

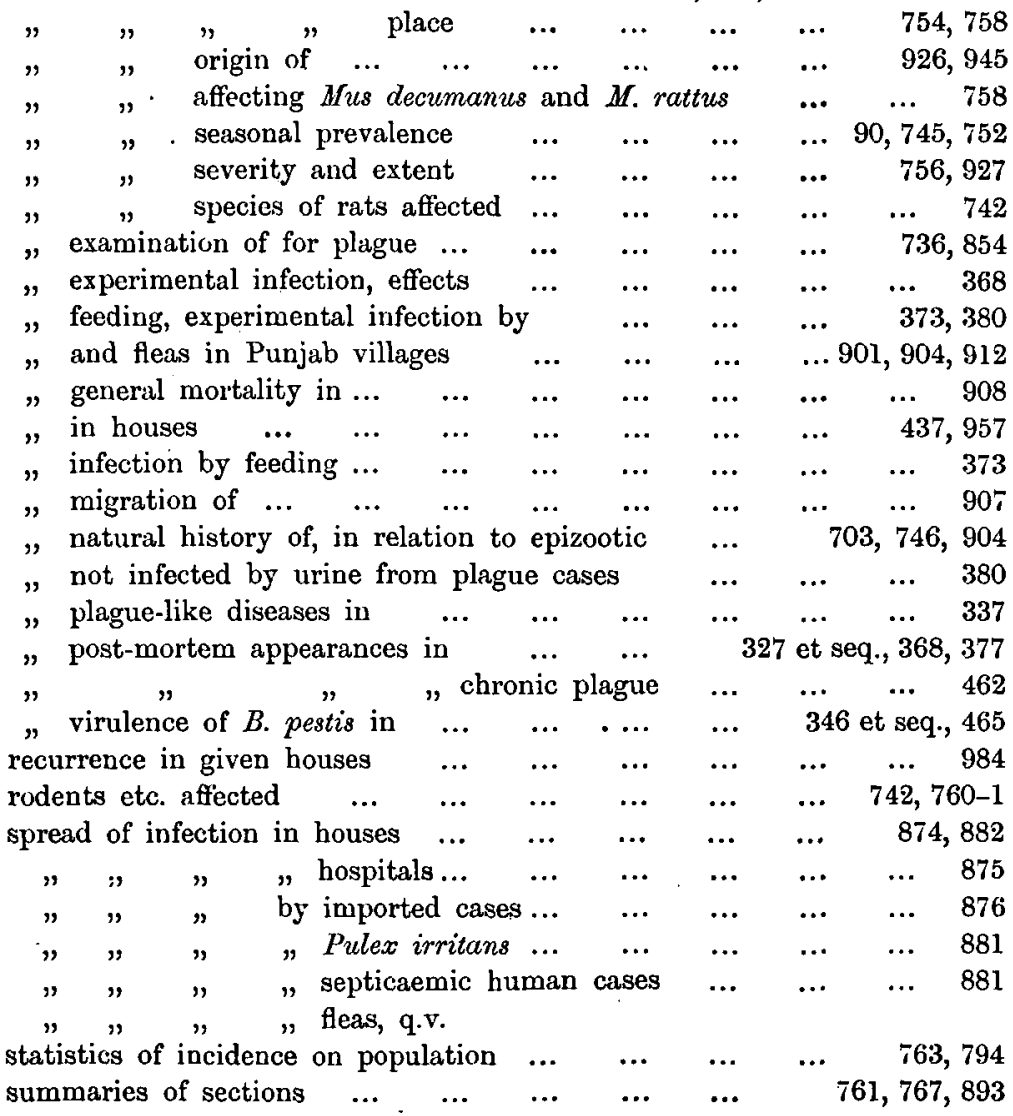


Plague transmission to rat by feeding ...

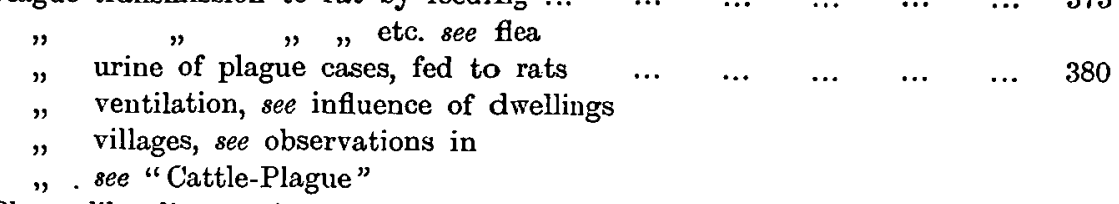

$\begin{array}{llllllllllll}\text { Plague-like diseases in rats } & \ldots & \ldots & \ldots & \ldots & \ldots & \ldots & \ldots & 337\end{array}$

$\begin{array}{llllllllll}\text { Polyneuritis gallinarum } & \ldots & \ldots & \ldots & \ldots & \ldots & \ldots & \ldots & 619 ; 634\end{array}$

Poultry, see Ship-beri-beri

Protozoa, see Piroplasma, Trypanosoma

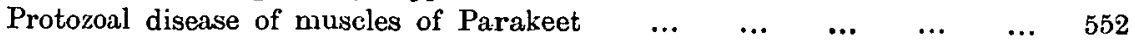

$\begin{array}{llllllllll}\text { Publications received } & \ldots & \ldots & \ldots & \ldots & \ldots & \ldots & \ldots & 161,686\end{array}$

Pulex, see under Plague flea

Rat, see Plague

Rheumatism, acute, see Meteorological

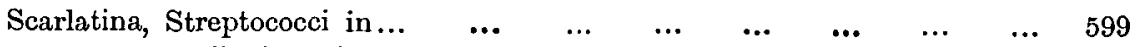

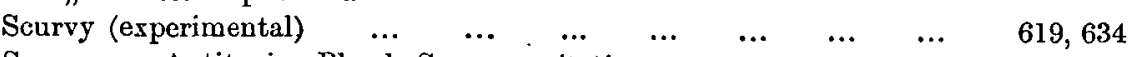

Serum, see Antitoxin, Blood, Supersensitation
Serum-disease, see Supersensitation

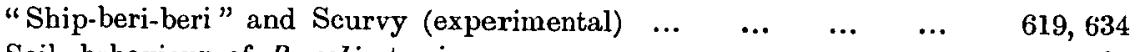

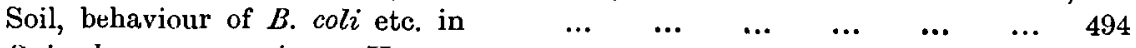

$\begin{array}{llllllllll}\text { Spirochata pertenuis, see Yaws } & & & & & & & & & \\ \text { Streptococci in scarlatina } & \ldots & \ldots & \ldots & \ldots & \ldots & \ldots & \ldots & \ldots & 599\end{array}$

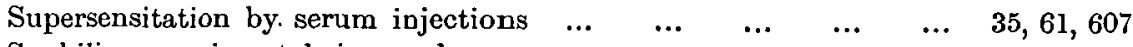

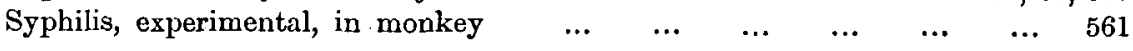

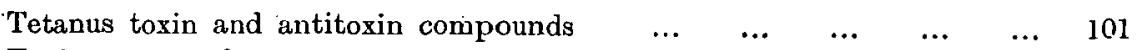

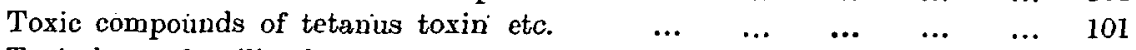

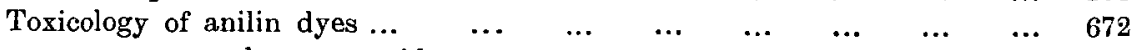

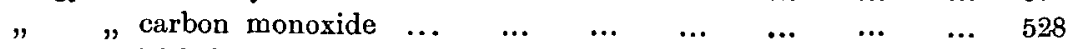

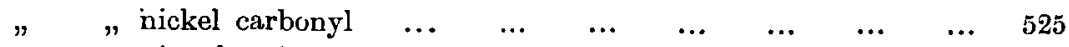

$\begin{array}{lllllllllll} & \text {, nitro-benzine } & \ldots & \ldots & \ldots & \ldots & \ldots & \ldots & \ldots & 672\end{array}$

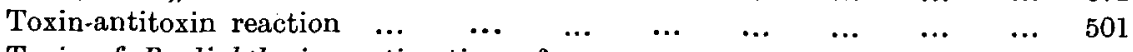

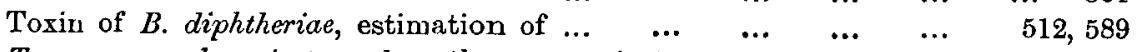

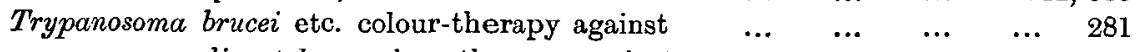

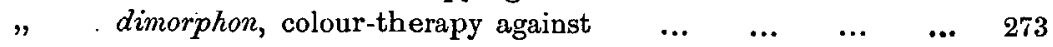

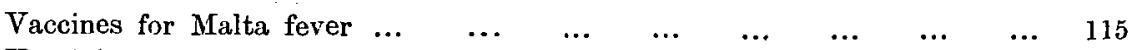

Vaccinia in animals, effect of light upon $\quad \begin{array}{lllllll}\ldots & \ldots & \ldots & \ldots & \ldots & 155\end{array}$

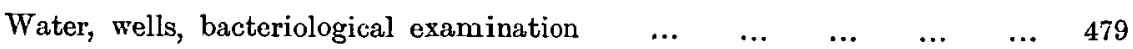

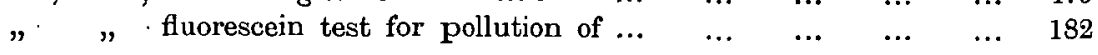

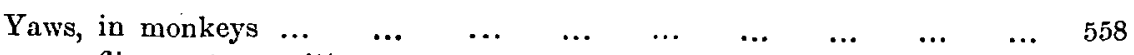

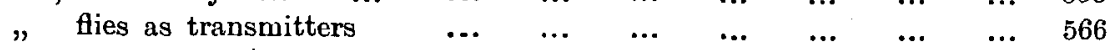

CAMBRIDGE : PRIN'TED BY JOHN CLAY, M.A. AT THE UNIVERSTTY PRESS. 